\title{
ON THE REALIZATION OF BAND-PASS TYPE SYSTEMS FOR BOUNDED BANDLIMITED SIGNALS
}

\author{
Holger Boche and Ullrich J. Mönich \\ Technische Universität Berlin, Heinrich-Hertz-Chair for Mobile Communications \\ Einsteinufer 25, 10578 Berlin, Germany
}

\begin{abstract}
In this paper we analyze band-pass type systems that operate on bounded bandlimited signals. For a very general class of band-pass type systems, we prove that there exists no linear realization of the systems in this class. Since ideal band-pass type systems are included in this class, it follows that there exists no linear realization of ideal band-pass type systems. This result is obtained under very general assumptions. For example, we do not assume the systems to be time-invariance. Finally, it is shown that a non-linear realization of band-pass type systems is possible.
\end{abstract}

Index Terms - Low-pass, band-pass, system, stable, linear realization

\section{INTRODUCTION}

Filters are a widely used tool in signal processing and system theory. The filtering of signals is especially descriptive when the signals are treated in the frequency domain. Loosely speaking, filters can be used to extract certain frequency portions from the signal, while other frequencies are suppressed. The passband of a filter is a certain frequency interval that specifies the behavior of the filter: All signals that have only frequencies within the passband are not disturbed by the filter. Filters can be characterized according to their passband. It is common to distinguish between low-pass type, highpass type, and band-pass type filters. In the following we use the term system instead of filter because filters are often assumed to be linear and time-invariant, and we do not want to restrict our analysis a priori to systems with those properties.

Low-pass type, high-pass type, and band-pass type systems are also frequently used in theoretical analyses. In [1] for example, Logan develops a theory of modulation systems, in which band-pass type systems play a central role. Modulation systems are often based on the Hilbert transform. However, for general bounded bandlimited signals the Hilbert transform does not necessarily exist. Interestingly, for every bounded band-pass signal the Hilbert transform exists and is bounded [2].

For several reasons band-pass type systems should be efficient in the sense that

P1) every output signal has only frequencies within the passband,

P2) every input signal that has only frequencies within the passband is not disturbed by the system, and

P3) the system is stable.

Many applications require such an efficient system. For example in mobile communications the transmitted signal must not have any frequency content outside some predefined band, because otherwise it could interfere with other services that use these frequencies. Hence the used band-pass type system needs to fulfill property $\mathrm{P} 1$ ). On the other hand the desired signal within the passband should not be disturbed. This is what property P2) expresses. In property P3) we mean with stability that the norm of the output signal can be controlled.

In an abstract view, an efficient system is an operator that maps every input signal to an output signal in accordance with the properties P1)-P3) above. It is important to distinguish between the abstract concept of a system, or more general of an operator, and the actual realization. An operator can have many possible realizations with different properties. Take for example the differential operator, operating on the space of bounded bandlimited signals that vanish at infinity. One realization is given by

$$
f^{\prime}(t)=\pi \sum_{k=-\infty}^{\infty} f(k)\left(\frac{\cos (\pi(t-k))}{\pi(t-k)}-\frac{\sin (\pi(t-k))}{(\pi(t-k))^{2}}\right)
$$

This series is formally obtained from the Shannon sampling series

$$
f(t)=\sum_{k=-\infty}^{\infty} f(k) \frac{\sin (\pi(t-k))}{\pi(t-k)}
$$

by differentiating termwise. Another possible realization, which is obtained by differentiating the Valiron interpolation series [3, p. 12]

$$
\begin{aligned}
f(t)=f(0)+f^{\prime}(0) & \frac{\sin (\pi t)}{\pi} \\
& +t \sum_{\substack{k=-\infty \\
k \neq 0}}^{\infty} \frac{f(k)-f(0)}{k} \frac{\sin (\pi(t-k))}{\pi(t-k)},
\end{aligned}
$$

is given by

$$
\begin{aligned}
f^{\prime}(t)= & \sum_{\substack{k=-\infty \\
k \neq 0}}^{\infty} \frac{f(k)}{\pi(t-k) k}[\sin (\pi(t-k)) \\
& \left.+\frac{t \cos (\pi(t-k)) \pi(t-k)-\sin (\pi(t-k))}{t-k}\right] \\
& +f(0) \frac{\cos (\pi t) \pi t-\sin (\pi t)}{\pi t^{2}}+f^{\prime}(0) \cos (\pi t),
\end{aligned}
$$

The two realization have different properties. The sum in the second realization (2), for example, is absolutely convergent, while the sum in the first realization (1) is not absolutely convergent. Thus, from the fact that one representation does not have a specific property we cannot conclude that there exist no representation with this property.

It is reasonable to call a band-pass type system ideal if, in addition to $\mathrm{P} 1)-\mathrm{P} 3$ ) it has the property that

P4) the system output is the zero signal for every signal that has no frequencies within the passband. 
This definitions corresponds to accepted definition of an ideal filter. Obviously, the class of ideal band-pass type systems is a subclass of the efficient band-pass type systems. Further properties which are often assumed are time-invariance and linearity. Both are important for practical implementations as well as theoretical considerations.

In this paper we analyze band-pass type systems operating on bounded bandlimited signals. These signals are important in all applications where the peak value of the signal has to be controlled. For example, in mobile communication systems the peak value of the transmission signals has to be bounded by some constant in order that the power amplifier does not overload and the signal is not clipped.

For bounded bandlimited signals the Fourier transform does not need to exist. Hence, we have to be careful when we use terms like "frequencies of a signal". However, it will turn out that-with the proper definitions - we can mathematically rigorously formulate the properties of an efficient band-pass type system, which were listed above in an intuitive way, for systems operating on bounded bandlimited signals.

The paper is organized as follows. In Section 2 we introduce some definitions. In Section 3 we prove that there exists no linear (not even a time-variant) realization of efficient band-pass type systems for the signal space of bounded bandlimited signals. As a consequence two operations, which are often used and whose existence is generally taken for granted, the extraction of frequency components and the splitting of a signal into two parts with disjoint spectrum, cannot be performed in a stable manner with a linear system. Moreover, in Section 4 we show that a non-linear system with the properties P1)-P3) can be realized. Since the results are counterintuitive, we give the full proofs of the theorems, except for a few elementary computations.

\section{NOTATION AND DEFINITIONS}

In order to continue, we need some notation and definitions. Let $\hat{f}$ denote the Fourier transform of a function $f . L^{p}(\mathbb{R}), 1 \leq p<\infty$, is the space of all $p$ th-power Lebesgue integrable functions on $\mathbb{R}$, with the usual norm $\|\cdot\|_{p}$, and $L^{\infty}(\mathbb{R})$ is the space of all functions for which the essential supremum norm $\|\cdot\|_{\infty}$ is finite.

For $\sigma>0$ let $\mathcal{B}_{\sigma}$ be the set of all entire functions $f$ with the property that for all $\epsilon>0$ there exists a constant $C(\epsilon)$ with $|f(z)| \leq$ $C(\epsilon) \exp ((\sigma+\epsilon)|z|)$ for all $z \in \mathbb{C}$. The Bernstein space $\mathcal{B}_{\sigma}^{\infty}$ consists of all functions in $\mathcal{B}_{\sigma}$, whose restriction to the real line is in $L^{\infty}(\mathbb{R})$. A signal in $\mathcal{B}_{\sigma}^{\infty}$ is called bandlimited to $\sigma$. For those signals, we have the following lemma, which is a consequence of the PhragménLindelöf principle [4, Lecture 6].

Lemma 1. Let $f \in \mathcal{B}_{\sigma}^{\infty}, \sigma>0$. Then we have

$$
|f(z)| \leq\|f\|_{\infty} \mathrm{e}^{\sigma|\operatorname{Im}(z)|}
$$

for all $z \in \mathbb{C}$.

For signals in $\mathcal{B}_{\sigma}^{\infty}$ the Fourier transform does not necessarily exist. This is the reason why we use the following definition to define bounded band-pass signals. For $\omega_{1}<\omega_{2}$ let $\mathcal{K}_{+}\left(\omega_{1}, \omega_{2}\right)$ denote the space of all functions $f \in L^{1}(\mathbb{R})$, whose Fourier transform fulfills $\hat{f}(\omega)=1$ for $\omega \in\left[\omega_{1}, \omega_{2}\right]$. The space $\mathcal{B}_{+\left[\omega_{1}, \omega_{2}\right]}$ consists of all signals $f \in L^{\infty}(\mathbb{R})$ that fulfill $f(t)=\int_{-\infty}^{\infty} f(\tau) K(t-\tau) \mathrm{d} \tau$ for all $t \in \mathbb{R}$ and all $K \in \mathcal{K}_{+}\left(\omega_{1}, \omega_{2}\right)$. For $0 \leq \omega_{1}<\omega_{2}<\infty$ let $\mathcal{K}\left(\omega_{1}, \omega_{2}\right)$ denote the space of all functions $f \in L^{1}(\mathbb{R})$, whose Fourier transform fulfills $\hat{f}(\omega)=1$ for $|\omega| \in\left[\omega_{1}, \omega_{2}\right]$. The space
$\mathcal{B}_{\left[\omega_{1}, \omega_{2}\right]}^{\infty}$ consists of all signals $f \in L^{\infty}(\mathbb{R})$ that fulfill $f(t)=$ $\int_{-\infty}^{\infty} f(\tau) K(t-\tau) \mathrm{d} \tau$ for all $t \in \mathbb{R}$ and all $K \in \mathcal{K}\left(\omega_{1}, \omega_{2}\right)$. Note that we have $\mathcal{B}_{\omega_{2}}^{\infty}=\mathcal{B}_{\left[0, \omega_{2}\right]}^{\infty}=\mathcal{B}_{+\left[-\omega_{2} . \omega_{2}\right]}^{\infty}$, according to this definition.

\section{LINEAR REALIZATION OF EFFICIENT BAND-PASS TYPE SYSTEMS}

In this section we analyze whether a linear realization of efficient band-pass type systems exists for $\mathcal{B}_{\pi}^{\infty}$, i.e., the space of bounded bandlimited signals.

Theorem 1. Let $0 \leq \omega_{1}<\omega_{2} \leq \pi$ with $w_{2}-w_{1}<\pi$. There exists no linear operator $\bar{T}$ defined on $\mathcal{B}_{\pi}^{\infty}$ with the properties

i) $\operatorname{range}(T) \subseteq \mathcal{B}_{\left[\omega_{1}, \omega_{2}\right]}^{\infty}$ and

ii) $T f=f$ for all $f \in \mathcal{B}_{\left[\omega_{1}, \omega_{2}\right]}^{\infty}$.

iii) $T: \mathcal{B}_{\pi}^{\infty} \rightarrow \mathcal{B}_{\left[\omega_{1}, \omega_{2}\right]}^{\infty}$ is bounded.

The properties i)-iii) are the mathematical formulation of the properties P1)- P3), which determine an efficient system. Property i) means that the system output has only frequencies in the range $\left[-\omega_{2},-\omega_{1}\right] \cup\left[\omega_{1}, \omega_{2}\right]$. Property ii) says that every signal with frequencies concentrated in the passband is not disturbed by the system, and property iii) expresses the stability of the system. The additional condition $w_{2}-w_{1}<\pi$ in Theorem 1 was only included to prevent the case where $\omega_{1}=0$ and $\omega_{2}=\pi$. In this case the identity operator would trivially fulfill the properties i), ii), and iii).

Theorem 1 shows that there exists no linear operator defined on $\mathcal{B}_{\pi}^{\infty}$ with the properties i)-iii). Consequently, a linear realization of efficient band-pass type systems for the signal space $\mathcal{B}_{\pi}^{\infty}$ cannot exist. Since the class of ideal band-pass type systems is a subclass of the efficient band-pass type systems, this implies that there exists no linear realization of ideal band-pass type systems.

Remark 1 . Since every low-pass type system can be seen as a bandpass type system with a passband that starts from zero, Theorem 1 also implies that there exists no linear realization of efficient lowpass type systems for the signal space $\mathcal{B}_{\pi}^{\infty}$.

Note that the result of Theorem 1 is very general, because there are many conceivable realizations. For example we do not restrict the systems to be time-invariant.

Proof of Theorem 1. The proof is divided into two parts. In the fist part we proof the assertion for $0 \leq \omega_{1}<\omega_{2}<\pi$ and in the second part for $0<\omega_{1}<\omega_{2}=\pi$.

First part: Let $\omega_{1}, \omega_{2} \in \mathbb{R}$ with $0 \leq \omega_{1}<\omega_{2}<\pi$ be arbitrary but fixed. The non-existence is proved indirectly. We assume that there exists a bounded linear operator $T$ defined on $\mathcal{B}_{\pi}^{\infty}$ with the properties i) and ii) and construct a contradiction.

For the proof we need the functions

$$
f_{N}(t)=\frac{\mathrm{e}^{i \omega_{2} t}}{2 i} \sum_{k=1}^{\lfloor N \gamma\rfloor} \frac{1}{k}\left(\mathrm{e}^{i \pi \frac{k}{N} t}-\mathrm{e}^{-i \pi \frac{k}{N} t}\right), \quad N \in \mathbb{N},
$$

where

$$
\gamma=\min \left(\frac{\omega_{2}-\omega_{1}}{\pi}, 1-\frac{\omega_{2}}{\pi}\right)
$$

Thus, $f_{N} \in \mathcal{B}_{\pi}^{\infty}$ for all $N \in \mathbb{N}$. Moreover, let $f_{N, \tau}(t)=f_{N}(t+\tau)$. Then we have

$$
\left\|f_{N, \tau}\right\|_{\infty}=\left\|f_{N}\right\|_{\infty}=\left\|\sum_{k=1}^{\lfloor N \gamma\rfloor} \frac{\sin (\pi \cdot k / N)}{k}\right\|_{\infty} \leq C_{1}<\infty
$$




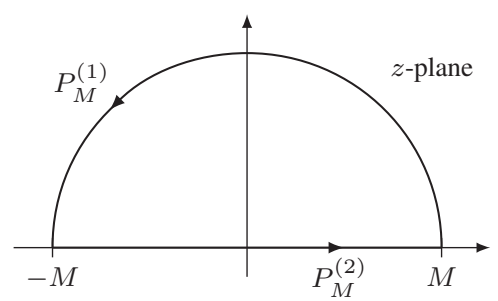

Fig. 1. Integration Paths $P_{M}^{(1)}$ and $P_{M}^{(2)}$ in the Complex Plane.

for all $N \in \mathbb{N}$ and all $\tau \in \mathbb{R}$, where the constant $C_{1}$ is independent of $N$ [5, p. 183]. For the moment, let $N \in \mathbb{N}$ and $t \in \mathbb{R}$ be arbitrary but fixed. We have

$$
\begin{aligned}
\left(T f_{N, \tau}\right)( & t-\tau) \\
= & \frac{1}{2 i} \sum_{k=1}^{\lfloor N \gamma\rfloor} \frac{1}{k} \mathrm{e}^{i \tau\left(\omega_{2}+\frac{k}{N} \pi\right)} \underbrace{T\left[\mathrm{e}^{i \cdot\left(\omega_{2}+\frac{k}{N} \pi\right)}\right](t-\tau)}_{=u(k, \tau)} \\
& -\frac{1}{2 i} \sum_{k=1}^{\lfloor N \gamma\rfloor} \frac{1}{k} \mathrm{e}^{i \tau\left(\omega_{2}-\frac{k}{N} \pi\right)} T\left[\mathrm{e}^{i \cdot\left(\omega_{2}-\frac{k}{N} \pi\right)}\right](t-\tau) .
\end{aligned}
$$

Since $\omega_{2}-\frac{k}{N} \pi \geq \omega_{1}$ for all $1 \leq k \leq\lfloor N \gamma\rfloor$, the function $\mathrm{e}^{i \cdot\left(\omega_{2}-\frac{k}{N} \pi\right)}$ is in $\mathcal{B}_{\left[\omega_{1}, \omega_{2}\right]}^{\infty}$ and it follows that

$$
T\left[\mathrm{e}^{i \cdot\left(\omega_{2}-\frac{k}{N} \pi\right)}\right](t-\tau)=\mathrm{e}^{i(t-\tau)\left(\omega_{2}-\frac{k}{N} \pi\right)}
$$

for all $1 \leq k \leq\lfloor N \gamma\rfloor$, according to property ii), which is assumed to be true. Further, we know from i) that $u(k, \cdot) \in \mathcal{B}_{\omega_{2}}^{\infty}$ for $1 \leq$ $k \leq\lfloor N \gamma\rfloor$. Consequently, we obtain

$$
\begin{aligned}
\left(T f_{N, \tau}\right)(t-\tau)= & \frac{1}{2 i} \sum_{k=1}^{\lfloor N \gamma\rfloor} \frac{1}{k} \mathrm{e}^{i \tau\left(\omega_{2}+\frac{k}{N} \pi\right)} u(k, \tau) \\
& -\frac{1}{2 i} \sum_{k=1}^{\lfloor N \gamma\rfloor} \frac{1}{k} \mathrm{e}^{i \tau\left(\omega_{2}-\frac{k}{N} \pi\right)} \mathrm{e}^{i(t-\tau)\left(\omega_{2}-\frac{k}{N} \pi\right)} \\
= & \frac{1}{2 i} \sum_{k=1}^{\lfloor N \gamma\rfloor} \frac{1}{k} \mathrm{e}^{i \tau\left(\omega_{2}+\frac{k}{N} \pi\right)} u(k, \tau) \\
& -\frac{1}{2 i} \sum_{k=1}^{\lfloor N \gamma\rfloor} \frac{1}{k} \mathrm{e}^{i t\left(\omega_{2}-\frac{k}{N} \pi\right)}
\end{aligned}
$$

and

$$
\begin{aligned}
& \frac{1}{2 M} \int_{-M}^{M}\left(T f_{N, \tau}\right)(t-\tau) \mathrm{d} \tau \\
& =\frac{1}{2 i} \sum_{k=1}^{\lfloor N \gamma\rfloor} \frac{1}{k} \frac{1}{2 M} \int_{-M}^{M} \mathrm{e}^{i \tau\left(\omega_{2}+\frac{k}{N} \pi\right)} u(k, \tau) \mathrm{d} \tau \\
& \quad-\frac{1}{2 i} \sum_{k=1}^{\lfloor N \gamma\rfloor} \frac{1}{k} \mathrm{e}^{i t\left(\omega_{2}-\frac{k}{N} \pi\right)} .
\end{aligned}
$$

Next, we treat the integral on the right-hand side of (3) for $1 \leq k \leq$ $\lfloor N \gamma\rfloor$. According to Cauchy's integral theorem, we have

$$
\oint_{P_{M}^{(1)} \cup P_{M}^{(2)}} \mathrm{e}^{i z\left(\omega_{2}+\frac{k}{N} \pi\right)} u(k, z) \mathrm{d} z=0 .
$$

Since $u(k, \cdot) \in \mathcal{B}_{\omega_{2}}^{\infty}$, Lemma 1 guarantees the existence of a constant $C_{2}(k)$ such that

$$
|u(k, z)| \leq C_{2}(k) \mathrm{e}^{\omega_{2}|\operatorname{Im}(z)|}
$$

for all $z \in \mathbb{C}$, and it follows that

$$
\begin{aligned}
& \left|\frac{1}{2 M} \int_{-M}^{M} \mathrm{e}^{i \tau\left(\omega_{2}+\frac{k}{N} \pi\right)} u(k, \tau) \mathrm{d} \tau\right| \\
& =\left|\frac{1}{2 M} \int_{P_{M}^{(1)}} \mathrm{e}^{i z\left(\omega_{2}+\frac{k}{N} \pi\right)} u(k, z) \mathrm{d} z\right| \\
& \leq \frac{1}{2} \int_{0}^{\pi}\left|\mathrm{e}^{i M \mathrm{e}^{i \phi}\left(\omega_{2}+\frac{k}{N} \pi\right)}\right|\left|u\left(k, M \mathrm{e}^{i \phi}\right)\right| \mathrm{d} \phi \\
& \leq \frac{C_{2}(k)}{2} \int_{0}^{\pi} \mathrm{e}^{-M \sin (\phi)\left(\omega_{2}+\frac{k}{N} \pi\right)} \mathrm{e}^{\omega_{2} M \sin (\phi)} \mathrm{d} \phi \\
& =\frac{C_{2}(k)}{2} \int_{0}^{\pi} \mathrm{e}^{-\frac{k}{N} M \sin (\phi)} \mathrm{d} \phi .
\end{aligned}
$$

Bearing in mind that

$$
\int_{0}^{\pi / 2} \mathrm{e}^{-\frac{k}{N} M \sin (\phi)} \mathrm{d} \phi=\int_{\pi / 2}^{\pi} \mathrm{e}^{-\frac{k}{N} M \sin (\phi)} \mathrm{d} \phi
$$

and that $\sin (\phi) \geq 2 \phi / \pi$ for all $\phi \in[0, \pi / 2]$, we can further evaluate the integral on the right-hand side of (4):

$$
\begin{aligned}
\frac{C_{2}(k)}{2} \int_{0}^{\pi} \mathrm{e}^{-\frac{k}{N} M \sin (\phi)} \mathrm{d} \phi & \leq C_{2}(k) \int_{0}^{\pi / 2} \mathrm{e}^{-\frac{2 k M}{N \pi} \phi} \mathrm{d} \phi \\
& =C_{2}(k) \frac{N \pi}{2 k M}\left(1-\mathrm{e}^{-\frac{k M}{N}}\right) .
\end{aligned}
$$

Thus, we obtain

$\left|\frac{1}{2 M} \int_{-M}^{M} \mathrm{e}^{i \tau\left(\omega_{2}+\frac{k}{N} \pi\right)} u(k, \tau) \mathrm{d} \tau\right| \leq C_{2}(k) \frac{N \pi}{2 k M}\left(1-\mathrm{e}^{-\frac{k M}{N}}\right)$, and consequently

$$
\lim _{M \rightarrow \infty} \frac{1}{2 M} \int_{-M}^{M} \mathrm{e}^{i \tau\left(\omega_{2}+\frac{k}{N} \pi\right)} u(k, \tau) \mathrm{d} \tau=0
$$

for all $1 \leq k \leq\lfloor N \gamma\rfloor$. Using (5), it follows from (3) that

$$
\lim _{M \rightarrow \infty}\left|\frac{1}{2 M} \int_{-M}^{M}\left(T f_{N, \tau}\right)(t-\tau) \mathrm{d} \tau\right|=\left|\frac{1}{2} \sum_{k=1}^{\lfloor N \gamma\rfloor} \frac{1}{k} \mathrm{e}^{i t\left(\omega_{2}-\frac{k}{N} \pi\right)}\right| .
$$

Since $T$ is assumed to be a bounded linear operator, we have $\|T\|=$ $\sup _{\|f\|_{\mathcal{B} \infty} \leq 1}\|T f\|_{\infty}<\infty$, and $\|T f\|_{\infty} \leq\|T\|\|f\|_{\infty}$ for all $f \in \mathcal{B}_{\pi}^{\infty}$. It follows that

$$
\begin{aligned}
\left|\frac{1}{2 M} \int_{-M}^{M}\left(T f_{N, \tau}\right)(t-\tau) \mathrm{d} \tau\right| & \leq \frac{1}{2 M} \int_{-M}^{M}\left|\left(T f_{N, \tau}\right)(t-\tau)\right| \mathrm{d} \tau \\
& \leq\left\|T f_{N, \tau}\right\|_{\infty} \leq\|T\|\left\|f_{N, \tau}\right\|_{\infty} \\
& \leq\|T\| C_{1}<\infty .
\end{aligned}
$$

Thus, from (6) and (7), we obtain

$$
\left|\frac{1}{2} \sum_{k=1}^{\lfloor N \gamma\rfloor} \frac{1}{k} \mathrm{e}^{i t\left(\omega_{2}-\frac{k}{N} \pi\right)}\right| \leq\|T\| C_{1}<\infty
$$


which is valid for all $N \in \mathbb{N}$ and $t \in \mathbb{R}$, because $N$ and $t$ were arbitrary. On the other hand, for $t=0$ we have

$$
\begin{aligned}
\lim _{N \rightarrow \infty}\left|\frac{1}{2} \sum_{k=1}^{\lfloor N \gamma\rfloor} \frac{1}{k} \mathrm{e}^{i t\left(\omega_{2}-\frac{k}{N} \pi\right)}\right| & =\lim _{N \rightarrow \infty} \frac{1}{2} \sum_{k=1}^{\lfloor N \gamma\rfloor} \frac{1}{k} \\
& \geq \frac{1}{2} \lim _{N \rightarrow \infty} \log (N \gamma) \\
& =\infty,
\end{aligned}
$$

which is a contradiction.

Second part: Let $\omega_{1} \in \mathbb{R}$ with $0<\omega_{1}<\omega_{2}=\pi$ be arbitrary but fixed, and assume that there is a bounded linear operator $T$ defined on $\mathcal{B}_{\pi}^{\infty}$ with the properties i) and ii). Let $M_{\sigma}, \sigma \in \mathbb{R}$, denote the operator $\left(M_{\sigma} f\right)(t)=\mathrm{e}^{i \sigma t} f(t)$. Furthermore, let $k_{1}$ be a function in $L^{1}(\mathbb{R})$ with $\hat{k}_{1}(\omega)=1$ for $\omega_{1} \leq \omega \leq \pi$, and define the operator

$$
\left(T_{1} f\right)(t)=\left(f * k_{1}\right)(t)=\int_{-\infty}^{\infty} f(\tau) k_{1}(t-\tau) \mathrm{d} \tau .
$$

It can be shown that $T_{1} f \in \mathcal{B}_{+\left[\omega_{1}, \pi\right]}^{\infty}$. Moreover, it follows from (8) that

$$
\left\|T_{1} f\right\|_{\infty} \leq\|f\|_{\infty}\left\|k_{1}\right\|_{L^{1}(\mathbb{R})} .
$$

Thus, $T_{1}: \mathcal{B}_{\left[\omega_{1}, \pi\right]}^{\infty} \rightarrow \mathcal{B}_{+\left[\omega_{1}, \pi\right]}^{\infty}$ is a bounded operator. Finally, we need the operator $U=M_{-\pi} T_{1} T M_{\pi}$. Clearly, $U$ is a bounded linear operator that maps $\mathcal{B}_{+[-\pi, 0]}^{\infty}$ onto $\mathcal{B}_{+\left[\omega_{1}-\pi, 0\right]}^{\infty}$. For all $f \in$ $\mathcal{B}_{+\left[\omega_{1}-\pi, 0\right]}^{\infty}$ we have $T_{1} T M_{\pi} f=M_{\pi} f$ and consequently $U f=$ $M_{-\pi} T_{1} T M_{\pi} f=M_{-\pi} M_{\pi} f=f$. We can use the same proof technique as in part 1 to show that such an operator cannot exist.

\section{NON-LINEAR REALIZATION OF EFFICIENT BAND-PASS TYPE SYSTEMS}

In this section we drop the condition that the system is linear. The following theorem shows that a non-linear realization of efficient band-pass type systems is possible for the space $\mathcal{B}_{\pi}^{\infty}$.

Theorem 2. Let $0 \leq \omega_{1}<\omega_{2} \leq \pi$. There exists an operator $T$ defined on $\mathcal{B}_{\pi}^{\infty}$ with the properties

$$
\begin{aligned}
& \text { i) } \operatorname{range}(T) \subseteq \mathcal{B}_{\left[\omega_{1}, \omega_{2}\right]}^{\infty} \\
& \text { ii) } T f=f \text { for all } f \in \mathcal{B}_{\left[\omega_{1}, \omega_{2}\right]}^{\infty} \text {, and } \\
& \text { iii) }\|T f\|_{\infty} \leq 2\|f\|_{\infty} \text { for all } f \in \mathcal{B}_{\pi}^{\infty} \text {. }
\end{aligned}
$$

Although Theorem 2 shows that a non-linear realization of efficient band-pass type systems is possible for the space $\mathcal{B}_{\pi}^{\infty}$, it makes no statement whether an ideal band-pass type system can be realized.

Proof. Let $f \in \mathcal{B}_{\pi}^{\infty} \backslash \mathcal{B}_{\left[\omega_{1}, \omega_{2}\right]}^{\infty}$ be arbitrary but fixed, and consider

$$
C\left(f, \omega_{1}, \omega_{2}\right)=\inf _{h \in \mathcal{B}_{\left[\omega_{1}, \omega_{2}\right]}^{\infty}}\|f-h\|_{\infty} .
$$

It follows that there exists a sequence $\left\{g_{n}\right\}_{n \in \mathbb{N}} \subset \mathcal{B}_{\left[\omega_{1}, \omega_{2}\right]}^{\infty}$ such that

$$
\lim _{n \rightarrow \infty}\left\|f-g_{n}\right\|_{\infty}=C\left(f, \omega_{1}, \omega_{2}\right) .
$$

Moreover, since $\left\|g_{n}\right\|_{\infty} \leq\left\|f-g_{n}\right\|_{\infty}+\|f\|_{\infty}$, there exists a natural number $n_{0}$ such that $\left\|g_{n}\right\|_{\infty} \leq\left(1+C\left(f, \omega_{1}, \omega_{2}\right)\right)+\|f\|_{\infty}$ for all $n \geq n_{0}$. Thus, we can use Lemma 1 to obtain

$$
\begin{aligned}
\left|g_{n}(z)\right| & \leq\left\|g_{n}\right\|_{\infty} \mathrm{e}^{\omega_{2}|\operatorname{Im}(z)|} \\
& \leq\left[\left(1+C\left(f, \omega_{1}, \omega_{2}\right)\right)+\|f\|_{\infty}\right] \mathrm{e}^{\omega_{2}|\operatorname{Im}(z)|}
\end{aligned}
$$

for all $z \in \mathbb{C}$ and $n \geq n_{0}$. From Montel's theorem [6, p. 195] it follows that there exists an entire function $g$ and a subsequence $\left\{n_{k}\right\}_{k \in \mathbb{N}}$ such that $\lim _{k \rightarrow \infty} g_{n_{k}}(z)=g(z)$ for all $z \in \mathbb{C}$. Further, it can be shown that $g \in \mathcal{B}_{\left[\omega_{1}, \omega_{2}\right]}^{\infty}$. Since $f \in \mathcal{B}_{\pi}^{\infty} \backslash \mathcal{B}_{\left[\omega_{1}, \omega_{2}\right]}^{\infty}$ was arbitrary, we can define an operator $T_{1}: \mathcal{B}_{\pi}^{\infty} \backslash \mathcal{B}_{\left[\omega_{1}, \omega_{2}\right]}^{\infty} \rightarrow$ $\mathcal{B}_{\left[\omega_{1}, \omega_{2}\right]}^{\infty}, f \mapsto g$. On the other hand, for $f \in \mathcal{B}_{\left[\omega_{1}, \omega_{2}\right]}^{\infty}$ we define the operator $T_{2}: \mathcal{B}_{\left[\omega_{1}, \omega_{2}\right]}^{\infty} \rightarrow \mathcal{B}_{\left[\omega_{1}, \omega_{2}\right]}^{\infty}, f \mapsto f$. Next, we show that the operator $T: \mathcal{B}_{\pi}^{\infty} \rightarrow \mathcal{B}_{\left[\omega_{1}, \omega_{2}\right]}^{\infty}$ given by

$$
T f= \begin{cases}T_{1} f & \text { if } f \in \mathcal{B}_{\pi}^{\infty} \backslash \mathcal{B}_{\left[\omega_{1}, \omega_{2}\right]}^{\infty} \\ T_{2} f & \text { if } f \in \mathcal{B}_{\left[\omega_{1}, \omega_{2}\right]}^{\infty}\end{cases}
$$

is the desired operator with the properties i)-iii). The properties i) and ii) are obviously fulfilled, due to the construction of the operator $T$. Moreover, the inequality in iii) is true for all $f \in \mathcal{B}_{\left[\omega_{1}, \omega_{2}\right]}^{\infty}$ because for these functions we have $\|T f\|_{\infty}=\|f\|_{\infty}$. It remains to prove the inequality in iii) for all $f \in \mathcal{B}_{\pi}^{\infty} \backslash \mathcal{B}_{\left[\omega_{1}, \omega_{2}\right]}^{\infty}$. For all $t \in \mathbb{R}$ we have $\left|f(t)-g_{n_{k}}(t)\right| \leq\left\|f-g_{n_{k}}\right\|_{\infty}$ and consequently

$$
\begin{aligned}
|f(t)-g(t)| & =\lim _{k \rightarrow \infty}\left|f(t)-g_{n_{k}}(t)\right| \\
& \leq \lim _{k \rightarrow \infty}\left\|f-g_{n_{k}}\right\|_{\infty} \\
& =C\left(f, \omega_{1}, \omega_{2}\right) .
\end{aligned}
$$

Taking the supremum on both sides of (11), we obtain

$$
\|f-g\|_{\infty} \leq C\left(f, \omega_{1}, \omega_{2}\right) .
$$

This implies that

$$
\|f-g\|_{\infty}=C\left(f, \omega_{1}, \omega_{2}\right),
$$

because $C\left(f, \omega_{1}, \omega_{2}\right)=\inf _{h \in \mathcal{B}_{\left[\omega_{1}, \omega_{2}\right]}^{\infty}}\|f-h\|_{\infty} \leq\|f-g\|_{\infty}$. Equality (12) together with the fact that $C\left(f, \omega_{1}, \omega_{2}\right) \leq\|f\|_{\infty}$ gives

$$
\begin{aligned}
\|g\|_{\infty} & \leq\|f-g\|_{\infty}+\|f\|_{\infty} \\
& =C\left(f, \omega_{1}, \omega_{2}\right)+\|f\|_{\infty} \\
& \leq 2\|f\|_{\infty},
\end{aligned}
$$

which completes the proof.

\section{REFERENCES}

[1] B. F. Logan, Jr., "Signals designed for recovery after clippingI. localization of infinite products," AT\&T Bell Laboratories Technical Journal, vol. 63, no. 2, pp. 261-285, February 1984.

[2] R. P. Boas, Jr., "Some theorems on Fourier transforms and conjugate trigonometric integrals," Transactions of the American Mathematical Society, vol. 40, no. 2, pp. 287-308, 1936.

[3] P. L. Butzer, W. Splettstößer, and R. L. Stens, "The sampling theorem and linear prediction in signal analysis," Jahresbericht der Deutschen Mathematiker-Vereinigung, vol. 90, no. 1, pp. 170, January 1988.

[4] Boris Yakovlevich Levin, Lectures on Entire Functions, AMS, 1996.

[5] A. Zygmund, Trigonometric Series, vol. I, Cambridge University Press, 3 edition, 2002.

[6] Robert Everist Greene and Steven G. Krantz, Function Theory of One Complex Variable, John Wiley \& Sons, Inc., 1997. 